\title{
BMJ Open Using the Functional Resonance Analysis Method to explore how elastic compression therapy is organised and could be improved from a multistakeholder perspective
}

\author{
Rachel Hellen Petra Schreurs (D) ,1,2 Manuela A Joore, ${ }^{3}$ Hugo ten Cate, ${ }^{2,4}$ \\ Arina J ten Cate-Hoek ${ }^{2,4}$
}

To cite: Schreurs RHP, Joore MA, ten Cate $\mathrm{H}$, et al. Using the Functional Resonance Analysis Method to explore how elastic compression therapy is organised and could be improved from a multistakeholder perspective. BMJ Open 2021;11:e048331. doi:10.1136/ bmjopen-2020-048331

- Prepublication history and additional supplemental material for this paper are available online. To view these files, please visit the journal online (http://dx.doi.org/10.1136/ bmjopen-2020-048331).

Received 22 December 2020 Accepted 21 September 2021

Check for updates

(C) Author(s) (or their employer(s)) 2021. Re-use permitted under CC BY-NC. No commercial re-use. See rights and permissions. Published by BMJ.

For numbered affiliations see end of article.

Correspondence to Rachel Hellen Petra Schreurs; rachel.schreurs@mumc.nl

\section{ABSTRACT}

Objectives Elastic compression stocking (ECS) therapy is an important treatment for patients with deep venous thrombosis (DVT) and chronic venous insufficiency (CVI). This study aimed to provide insight into the structure and variability of the ECS therapy process, its effects on outcomes, and to elicit improvement themes from a multiple stakeholder perspective.

Design Thirty semi-structured interviews with professionals and patients were performed. The essential functions for the process of ECS therapy were extracted to create two work-as-done models using the Functional Resonance Analysis Method (FRAM). These findings were used to guide discussion between stakeholders to identify improvement themes.

Setting Two regions in the Netherlands, region Limburg and region North-Holland, including an academic hospital and a general hospital and their catchment region.

Participants The interviewees were purposely recruited and included 25 healthcare professionals (ie, general practitioners, internists, dermatologists, nurses, doctor's assistants, occupational therapists, home care nurses and medical stocking suppliers) and 5 patients with DVT or CVI. Results Two FRAM models were created (one for each region). The variability of the functions and their effect on outcomes, as well as interdependencies between functions, were identified. These were presented in stakeholder meetings to identify the structure of the process and designated variable and uniform parts of the process and its outcomes. Ultimately, six improvement themes were identified: dissemination of knowledge of the entire process; optimising and standardising initial compression therapy; optimising timing to contact the medical stocking supplier (when oedema has disappeared); improving the implementation of assistive devices; harmonising follow-up duration for patients with CVI; personalising follow-up and treatment duration in patients with DVT.

Conclusions This study provided a detailed understanding of how ECS therapy is delivered in daily practice by describing major functions and variability in performances and elicited six improvement themes from a multistakeholder perspective.
Strengths and limitations of this study

- We used the Functional Resonance Analysis Method (FRAM) to obtain a deep insight into how elastic compression stocking therapy was organised and performed in daily practice.

- Important insight into practice variation, interdependencies among functions, and their effects on outcomes became apparent using semi-structured interviews and stakeholder meetings.

- The FRAM approach demonstrates how an interdisciplinary approach can be used to extract different perspectives and co-design of interventions.

- Inclusion of more participants might allow for the identification of more variability providing a wider range of perspectives.

- Observer bias might be present since the main interviewer had a medical background.

\section{INTRODUCTION}

Elastic compression stocking (ECS) therapy is the cornerstone for the prevention and treatment of post-thrombotic syndrome after deep venous thrombosis (DVT) and the major conservative treatment modality for patients with chronic venous insufficiency (CVI).$^{1-5}$ Practically, it was our impression that there is a substantial variation in how ECS therapy is implemented. We assume this to result in heterogeneous and suboptimal care. However, a good understanding of this assumed variability is lacking. This study aimed to provide insight into the variability of the process as well as interactions between healthcare professionals and patients concerning ECS therapy in daily practice and to elicit improvement themes from a multistakeholder perspective.

From the moment ECS therapy is indicated, patients first need initial compression therapy 
to reduce oedema and to bridge the time until the start of definitive ECS therapy, which has to be fitted and delivered by a medical stocking supplier. ${ }^{56}$ Choosing optimal ECS therapy appears to be challenging since several decisions have to be made regarding the ECS characteristics (for example, pressure class, stiffness and ECS type) to optimally match the patient's clinical characteristics. ${ }^{7-9}$ Patients then are expected to apply and remove their ECS themselves. However, a large proportion of both patients with DVT and CVI are non-compliant to ECS therapy. ${ }^{10} 11$ One of the reasons for non-compliance include difficulties putting ECS on and/or removing the ECS. ${ }^{12-16}$ Some patients need assistive devices to preserve self-reliance, and a smaller proportion of patients rely on home care to assist with the application and removal of the ECS. The proportion of patients needing home care for this indication is not well known. One study found that, in elderly and overweight patients with CVI, $14.6 \%$ of patients relied on home care to either put on and/or remove the ECS. ${ }^{17}$ A recent trial in patients with acute DVT showed that $16.1 \%$ of patients received home care, though this included patients who required home care for the initial compression phase. ${ }^{18}$ Finally, a decision regarding ECS therapy duration will be made during regular outpatient clinic visits (only in DVT), whereas ECS therapy is permanent for patients with CVI.

ECS therapy is a complex process, with various healthcare professionals and patients collaborate and constantly, purposely or not, adjust their performances to each other and the working conditions (for example, restrictions in time, information or resources). ${ }^{19}$ This complexity results in a process of dynamic performances and interactions between healthcare professionals that can lead to both desirable and unwanted or unexpected outcomes that can spread through the whole system. ${ }^{20}$ As a consequence, a difference exists between work-as-done (which represents how work is done in daily practice) and workas-imagined (which represents how work is supposed to be done as stated in clinical guidelines and protocols). ${ }^{21}$ These insights have created a growing awareness of the importance of both context and practical applicability of recommendations and guidelines to optimise care, ${ }^{22-25}$ rather than a focus on guideline characteristics. Therefore, establishing specific facilitators and targets for improvements and gaining insight into how ECS therapy is managed and performed in clinical practice are essential to optimise current care.

To date, studies concerning ECS therapy have mainly focused on the duration and efficacy of ECS therapy to prevent post-thrombotic syndromein the case ofDVT, ${ }^{1-42627}$ reasons for non-compliance to ECS therapy ${ }^{10}{ }^{16} 28$ quality of life ${ }^{29}$ and improvement of self-reliance ${ }^{30-32}$ for patients with either DVT or CVI. To the best of our knowledge, no empirical studies to date have addressed the entire process of ECS therapy with gaps, interdependencies and variability across settings. Only four studies have focused on specific parts of daily practice (prescription patterns and patients' perspectives): three on DVT 132833 and one on venous disease in general. ${ }^{34}$ These studies consistently show a lack of consensus and considerable variation in practice among physicians regarding timing of initiation of ECS, duration of therapy (in patients with DVT) and compression strength. However, in complex care as ECS therapy, it is especially important to assess the entire process.

A useful method to analyse complex sociotechnical systems is to use the Functional Resonance Analysis Method (FRAM). FRAM arrives from the relatively new safety-II perspective. Whereas the safety-I primarily focused on finding the source for failures and reasons for non-adherence to guidelines to improve care, this promising perspective focuses on what can be learnt from daily practice. ${ }^{35}$ FRAM thereby uses the idea of resonance arising from the variability of everyday performance, which proactively results in improvement themes to reduce unwanted variability and strengthen performances leading to desired results. ${ }^{21}$ It follows a stepwise approach to create a model that provides clear insight into work-asdone, including all variability in performance as well as interactions and the consequences thereof on outcomes. Ultimately, the FRAM determines whether, and how far, work-as-done corresponds with work-as-imagined. The method has been widely applied in many work fields (eg, aviation, railway and airway traffic management, and construction), but its implementation in healthcare research remains more limited. ${ }^{36-46}$

Hence, this study aims to provide an overview of how ECS therapy is organised and to define suggestions for improvement using FRAM. It is the first step in developing a general cross-domain protocol to improve ECS therapy for patients with either DVT or CVI, matching daily practice, and in adherence to guidelines and scientific evidence.

\section{METHODS}

\section{Setting and population}

The study was conducted in two regions in the Netherlands (Limburg and North-Holland). These settings were chosen considering their geographical spread (Limburg in the most southern part of the Netherlands and North-Holland in the northern part) and because in North-Holland several interventions to improve self-reliance in elastic compression therapy have already been implemented. The hospital settings differ too, with a general hospital in North-Holland (on two locations) and an academic hospital in Limburg. To access care and appropriate diagnostic processes in both settings, patients may present at a general practitioner's (GP) practice. After clinical assessment, the GP determines whether the patient can be managed in primary care for further diagnosis and treatment or whether secondary care assessment is necessary.

The scope of this study is to identify the complete process of ECS therapy, from the moment ECS is indicated until the moment ECS can be stopped, for patients with DVT and CVI (stadium C4 and C5 according to the Clinical-Etiological-Anatomical-Pathophysiological classification system). ${ }^{47}$ 


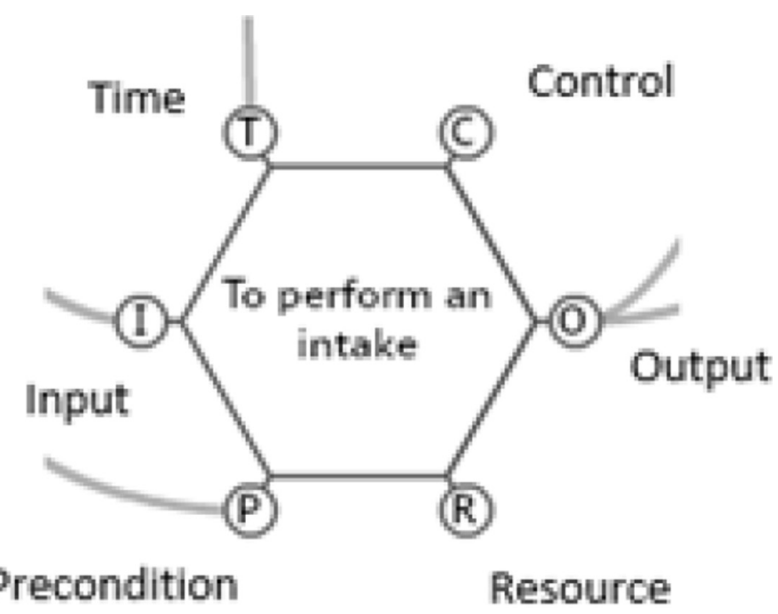

Figure 1 Functional Resonance Analysis Method activity hexagon. The six aspects represent: input (I): what the function activates, acts on or changes; time (T): any time constraints that might affect the function; control (C): how the function is monitored or controlled; output $(\mathrm{O})$ : the output or change of state that emerges from the function; resource $(\mathrm{R})$ : materials or people needed to carry out the function or materials consumed during the function; precondition $(\mathrm{P})$ : a condition that must be satisfied before the function can be started.

Stadium C6 patients (with an active venous ulcer) were excluded since these patients are generally not able to be self-reliant as they depend on home care for wound management. These patients are therefore expected to have fewer benefits from optimising care.

\section{Study design}

FRAM consists of four steps. The first step has a dual objective: first to explore work-as-imagined based on work instructions, guidelines and recent literature to globally identify the system's main activities (called functions) and the healthcare professionals who are involved; second, to visualise work-asdone and to systematically identify the functions that constitute ECS therapy in its entirety. These functions are identified based on interviews with involved healthcare professionals. Functions are divided into foreground functions (the system's main functions, whose variability may have consequences for its other functions) and background functions (those functions that are considered to be more stable and are on themselves not the focus of the current study, although they may introduce variability into the main functions). The functions are then visualised as hexagons using the FRAM Model Visualizer (FMV) software (V.0.4.1). ${ }^{48}$ Thereafter, the foreground functions are characterised by six aspects necessary to realise the function: input, output, precondition, resource, control and time (see figure 1).

The second step is to identify and characterise the variability in output of the individual functions in the FRAM model based on the information provided in the interviews. This output variability can be internal (variability in the function itself) or external (variability in working condition, that is, the conditions under which the function is performed). The third step is to identify how the aspects can connect different functions and identify interdependencies (also called couplings). For example, if the output of function A is also defined as a precondition for function $\mathrm{B}$, then these functions are coupled (a so-called 'upstream-downstream coupling') since variability in the output of function A impacts the performance of function $\mathrm{B}$. The variability in each function's output can be identified as well as its effect on the performance of other downstream functions (ie, functions performed later in the process). Finally, it is possible to develop recommendations on how to monitor and influence the variability by attenuating the variability that can lead to undesirable results or by enhancing the variability that can lead to desired results. ${ }^{49}$

\section{Data collection and analysis}

Identification of system functions and aspects (step 1)

To identify the system's main functions, a work-as-imagined model was created based on local protocols of the two selected regions, guidelines ${ }^{50-53}$ and recent literature. ${ }^{4}$ Healthcare professionals involved in the system were identified for each region and semi-structured interviews were planned to identify main functions and aspects for the work-as-done models. The interviewees were purposely recruited starting with a senior policy adviser from a home care organisation in Limburg, and a senior project manager from a home care organisation in North-Holland. Other experienced professionals were recruited through consecutive interviewees until data saturation was reached, defined as three consecutive interviews during which no new functions or output emerged. $^{54}$

The healthcare professionals were contacted by phone or email and all agreed to participate and consented verbally. One interviewer (RS) conducted the audiorecorded interviews with each interviewee either at the healthcare professional's workplace or the patient's home (Limburg) or by telephone (North-Holland). Interviews were guided by an interview template based on questions of the FRAM, in which the interviewer was trained before start of the interviews. ${ }^{49}$ Afterward, the interviews were transcribed verbatim. Follow-up questions were used to clarify any unclear descriptions and no repeat interviews were performed. A deeper understanding of the process was obtained from the interviews and the essential functions, including the six aspects of the function's hexagon required for ECS therapy, were extracted using an iterative modelling process to create the work-as-done models. Both the identified aspects and functions were discussed with two coauthors (AJtC-H, MJ) until consensus was reached. Subsequently, the functions and aspects were uploaded to the FMV software.

Assessment of variability of function output and interdependencies (steps 2 and 3)

To determine how the output variability of each function affected the process, functions were analysed based on whether they varied internally, externally or due to couplings reported in the interview data. Additionally, it was possible to consider variability between different 
healthcare professionals performing the same function in the process. Variability was primarily assessed by the interviewer (RS) and discussed with coauthors (AJtC-H, MJ) until consensus was reached. Afterwards, a list of potential improvement themes was compiled following a thematic analysis based on improvement suggestions from interviewees, the described variability in the interviews and the presumed effects on undesirable outcomes. This list was provided to all stakeholders before the discussion meeting.

Validation of the FRAM model and identification of opportunities for improvement to optimise current care (step 4)

A meeting of local key stakeholders, involving a selection of interviewees (one per discipline) as well as local policymakers, was organised for both regions separately. In these meetings, the work-as-done models were presented to reflect on the FRAM findings and validate the models. Responses from stakeholders were used to adjust the presented FRAM models. Thereafter, the FRAM models and the list of the potential improvement themes were used to reconcile improvement suggestions with work-asdone until consensus regarding the main improvement themes was reached. After the meetings, the adjusted models were sent to the stakeholders for final validation.

\section{Patient and public involvement}

Patients were viewed as equally important stakeholders and from the start of the project involved as interviewees $(n=5)$ in order to integrate their perspectives on work-asdone. Subsequently, their perspectives were included in the final FRAM models and improvement themes.

\section{RESULTS}

\section{FRAM model}

A total of 30 interviews: 15 face-to-face interviews for Limburg, and 15 telephonic interviews for North-Holland, were performed. Interviews lasted between 30 and $90 \mathrm{~min}$. The profiles of interviewees are listed in table 1 . Initially, separate models for DVT and CVI were created per region. Ultimately, as most functions for the DVT and CVI processes were equal, functions were combined and modelled in one FRAM model per region (figures 2 and 3). The Limburg model consisted of 14 foreground functions and the North-Holland model of 18. Four timeconsecutive phases were empirically identified from the interviews, which were similar for both models.

Phase 1, the initial compression therapy, includes identifying treatment indications; selecting the type of initial compression therapy; and implementing initial compression therapy either self-reliantly by the patient, provided by a caregiver at the outpatient clinic or general practice, or provided by home care nurses. Phase 2 is initiating ECS therapy. In this phase, the patient is expected to contact the medical stocking supplier who conducts an intake, a fitting consult and delivers the ECS. Subsequently, the medical stocking supplier estimates the patient's ability
Table 1 Number of interviewees per stakeholder group

\begin{tabular}{lll}
\hline Interviewees & $\begin{array}{l}\text { Limburg } \\
(\mathbf{1 5 )}\end{array}$ & $\begin{array}{l}\text { North-Holland } \\
\text { (15) }\end{array}$ \\
\hline General practitioners & 2 & 2 \\
\hline Internists & 2 & 3 \\
\hline Dermatologist & 1 & 1 \\
ER nurses & 2 & 1 \\
\hline Doctor's assistant & Not & 1 \\
dermatology & applicable & \\
\hline Nurse dermatology & 1 & Not applicable \\
\hline Medical stocking suppliers & 2 & 1 \\
Home care nurses & 2 & 2 \\
\hline $\begin{array}{l}\text { Occupational therapists } \\
\text { Patients with deep venous } \\
\text { thrombosis }\end{array}$ & 1 & 1 \\
\hline $\begin{array}{l}\text { Patients with chronic } \\
\text { venous insufficiency }\end{array}$ & 1 & 1 \\
\hline
\end{tabular}

ER, emergency room.

to be self-reliant (with or without an assistive device) and implements an assistive device if possible. If the medical stocking supplier estimates that it is not possible to implement the assistive device at that moment, downstream functions can be activated (eg, the occupational therapist for additional training) or the patient is advised to contact home care. In phase 3, some patients apply and remove their ECS self-reliantly, whereas others are trained to use the assistive device or home care is arranged to apply and remove the ECS. Phase 4 consists of maintenance ECS therapy and follow-up. A detailed description of the functions, the main aspects and output variability is outlined in the online supplemental tables 1 and 2 .

In both regions, the treating physician (ie, internist, dermatologist or GP) and the medical stocking supplier play key roles in the process. The treating physician (function 5 in the Limburg model and 4 and 8 in the North-Holland model) makes decisions regarding the type of initial compression therapy, affecting the course in downstream functions. Additionally, the treating physician generates prescriptions (eg, for multilayer compression bandages), instructions (eg, when to call the medical stocking supplier) and referrals (eg, for definitive ECS); documents that play important roles in downstream functions. Furthermore, the medical stocking supplier (function 9 in the Limburg model and 14 in North-Holland) provides outputs (implementing an assistive device, referring the patient to the occupational therapist or advising the patient to contact home care) affecting the patient's course in downstream functions. Finally, both models included downstream functions (ie, the follow-up appointments with the internist, the dermatologist or the GP, functions $14 \mathrm{a}-\mathrm{c}$ in the Limburg model and $18 \mathrm{a}+\mathrm{b}$ in the North-Holland model) that controlled the progress of the entire process. 


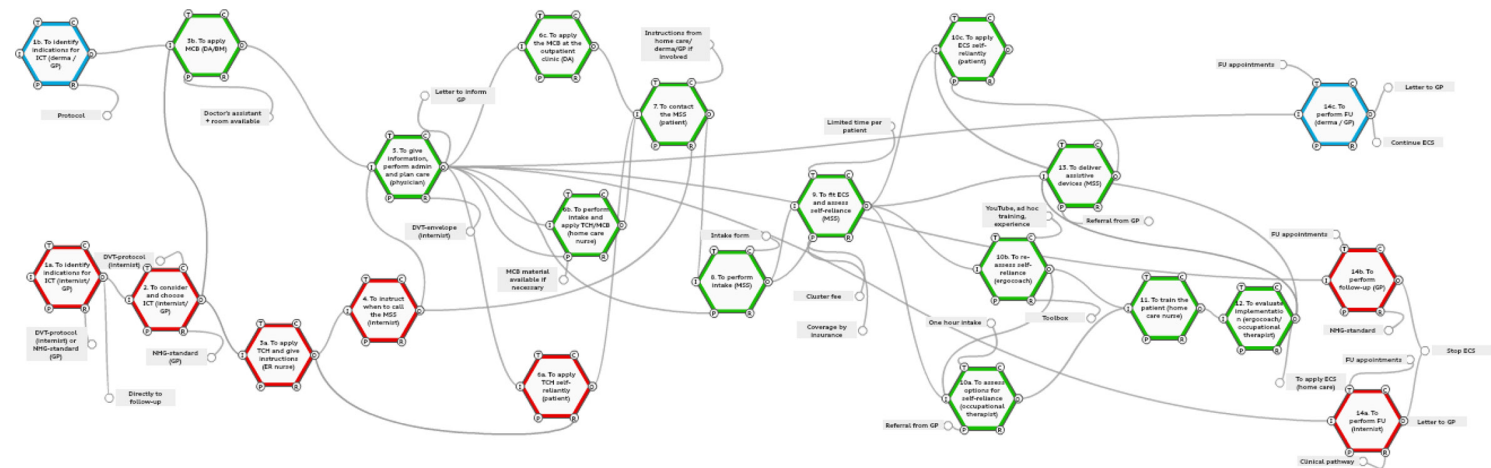

Figure 2 Functional Resonance Analysis Method model chronic venous insufficiency (CVI) and deep venous thrombosis (DVT) region Limburg. The red functions are specific functions for the process of DVT. The blue functions are specific functions for CVI. The green functions can occur in both processes of DVT and CVI, and activities and variability were observed to be the same for both DVT and CVI. DA, doctor's assistant; ECS, elastic compression stocking; ER, emergency room; FU, followup; GP, general practitioner; ICT, initial compression therapy; MCB, multilayer compression bandage; MSS, medical stocking supplier; NHG, Nederlands Huisartsen Genootschap (Dutch General Practitioner Society); TCH, temporary compression hosiery.

\section{Main themes for improvement}

In each region, a selection of potential improvement themes $(\mathrm{n}=11$ for Limburg and $\mathrm{n}=12$ for North-Holland, see table 2) was made to guide two meetings with stakeholders ( $\mathrm{n}=8$ for Limburg and $\mathrm{n}=9$ for North-Holland). Six major themes for improvement interventions were identified that were the same for both regions and are discussed in the next sections.

Theme 1: dissemination of knowledge of the entire process among all healthcare professionals involved

All stakeholders agreed that for the process to be optimised, instead of being knowledgeable only on the part in which they are directly involved, it is important that knowledge about the entire process is disseminated among all healthcare professionals involved. This is important to optimise the coordination among functions, provide uniform information to the patient and to involve the patient in the decision-making process at all phases of the process. This is especially the case for the frontline professionals working in phase 1 (initiating initial compression therapy), which enables them to better inform patients on the entire process at an early point in time and in a personalised manner.

Theme 2: optimising and standardising initial compression therapy for both patients with DVT and CVI

The FRAM showed that nearly all patients with DVT and CVI were treated with initial compression therapy, except for patients with DVT without oedema diagnosed by the GP in Limburg and patients with DVT diagnosed at one hospital location in North-Holland. At this hospital location, even definitive ECS was omitted in patients with DVT without complaints and no oedema. Logistics problems

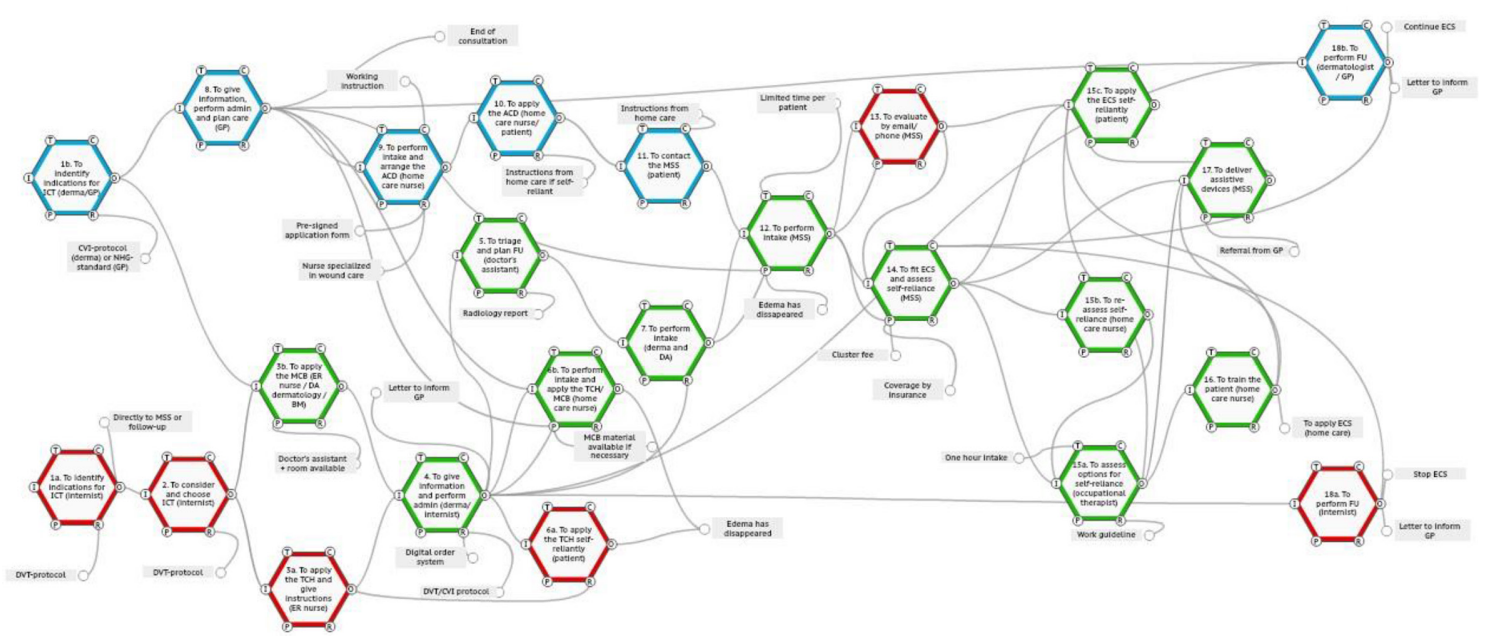

Figure 3 Functional Resonance Analysis Method model deep venous thrombosis (DVT) and chronic venous insufficiency (CVI) region North-Holland. The red functions are specific functions for the process of DVT. The blue functions are specific functions for CVI. The green functions can occur in both processes of DVT and CVI, and activities and variability were observed to be the same for both CVI and DVT. ACD, adjustable compression device; DA, doctor's assistant; ECS, elastic compression stocking; ER, emergency room; FU, follow-up; GP, general practitioner; ICT, initial compression therapy; MCB, multilayer compression bandage; MSS, medical stocking supplier; NHG, Nederlands Huisartsen Genootschap (Dutch General Practitioner Society); $\mathrm{TCH}$, temporary compression hosiery. 
Table 2 Selection of potentially improvement themes

\begin{tabular}{l} 
Region Limburg (11) \\
Deep venous thrombosis \\
To harmonise the advice to use initial and/or definitive ECS therapy \\
To harmonise the advised duration of ECS therapy \\
To increase knowledge of ER nurses regarding the instruction and demonstration (12) \\
of the TCH \\
Chronic venous insufficiency \\
poep organise follow-up and optimise patient knowledge on when to contact the treating physician \\
Deep venous thrombosis and chronic venous insufficiency \\
To optimise and harmonise initial compression therapy \\
To harmonise working methods for selecting and implementing assistive devices between medical stocking suppliers \\
To optimise the implementation of assistive devices in patients receiving home care for ECS therapy \\
To optimise the timing to contact the medical stocking supplier towards the end of the initial compression phase \\
To improve communication between medical stocking suppliers and home care nurses \\
To aim for uniformity in declaration requirements among insurance companies \\
\hline ECS, elastic compression stocking; ER, emergency room; TCH, temporary compression hosiery.
\end{tabular}

at the outpatient clinic and suboptimal awareness of the consequences of the omission of therapy were identified as the main drivers for these differences in approach. However, stakeholders agreed that initial and definitive compression therapy should be standardised for all patients with DVT, regardless of accompanying symptoms, to reduce the risk of post-thrombotic syndrome.

Further variation was observed regarding the type of initial compression therapy prescribed by GPs, dermatologists, and internists in both regions for both patients with DVT and CVI. Initial therapy included multilayer compression bandages, adjustable compression devices (only for patients with CVI from the GP in North-Holland) or temporary compression hosiery (only for patients with DVT in both regions). Although all treating physicians did use some kind of standardised methods to select initial compression therapy, these methods were not aligned among the different disciplines, which can have severe consequences for patients' chances to maintain self-reliance depending on the treating physician. Consensus was reached that in the context of preserving and promoting patients' self-reliance, adjustable compression devices or temporary compression hosiery should be considered as the preferred therapy instead of multilayer compression bandages for patients with DVT as well as patients with CVI. Additionally, stakeholders agreed that maintaining selfreliance early in the process can facilitate the success rate for implementation of assistive devices since it appeared more challenging to ensure self-reliance for the use of ECS if patients were already using home care.

Theme 3: optimising timing to contact the medical stocking supplier towards the end of the initial compression phase

In both regions, the exact timing of the intake at the medical stocking supplier was mentioned as a point for improvement. In an estimated $10 \%-30 \%$ of patients, oedema had not disappeared sufficiently at the time of intake, preventing the ECS to be fitted. Subsequently, a new appointment had to be made resulting in a delay in the downstream functions. Proposed strategies to guide this problem differed per region. One suggestion was that the speed and efficiency of the process could be further enhanced by providing better support to self-reliant patients on when to contact the medical stocking supplier. Another suggestion was to actively question the patient regarding the presence of oedema when the patient contacts the medical stocking supplier. Both suggestions were assessed to be practically feasible and were expected to help prevent unnecessary additional appointments and delays in the process.

Theme 4: improving the implementation of assistive devices to maintain patient's self-reliance

It was recognised by stakeholders that maintaining patient self-reliance and starting the implementation of assistive devices from the start of ECS therapy were key factors for increasing adherence to therapy.

The medical stocking supplier is initially responsible for estimating self-reliance abilities, and if necessary, select an assistive device and provide training. They perceive the assessment of patients' abilities, and the selection of a suitable assistive device challenging, in particular for elderly patients. Most importantly, since it has to be done in a short period (during the fitting consult, with an average duration of $10-15 \mathrm{~min}$ ); the assessment of a patient's abilities at this point is essential because if patients eventually turn out to be less self-reliant as previously expected, the expense of the assistive device will not be covered by the insurance company. Additionally, most elderly patients require additional time to be trained in 
the use of the assistive device. Furthermore, the cluster fee that medical stocking suppliers receive is a set amount that will not be increased if additional activities are performed to keep patients self-reliant. For either of these reasons, medical stocking suppliers can decide to not select and implement an assistive device themselves but instead refer patients to the occupational therapist or advise patients to contact the home care organisation to apply and remove the ECS.

Home care nurses mentioned that, in their experience, there is a low threshold for referral to the home care organisation, which probably results in more patients than necessary, using home care. This is an important concern as the home care organisations are already facing a high and increasing workload due to the ageing population and subsequent higher need for home care, which becomes hard to manage. Both home care organisations developed solutions to reduce the proportion of patients requiring home care for ECS therapy. In North-Holland, home care nurses reassess the patient's ability for self-reliance and refer selected patients to the occupational therapist to select and train an assistive device when indicated. In Limburg, specialised home care nurses select and implement assistive devices themselves. However, this adds to the workload of home care nurses, and the proportion of patients who become self-reliant following the intervention through home care nurses was noted to be low.

\section{Theme 5: harmonising follow-up duration for patients with CVI} In patients with CVI, there was variability in conducting follow-up appointments among GPs, and between dermatologists and GPs in both regions. Most GPs mentioned that they did not plan any follow-up appointments but relied on the patient's responsibility to alert them if problems occurred, whereas dermatologists standardised a follow-up appointment after 6 weeks. Dermatologists emphasised the importance of a prespecified follow-up appointment to repeat information regarding treatment goals and adherence to ECS therapy and to estimate the patient's risk for future problems. Ultimately, consensus was reached that follow-up appointments for patients with CVI are essential.

\section{Theme 6: personalising follow-up and treatment duration in patients with DVT}

In patients with DVT, considerable variations were found in both follow-up frequency and duration (6 weeks-24 months), as well as the advice when to stop ECS therapy among internists between the two hospital locations in North-Holland and between internists and GPs in both regions. In Limburg, internists gave individualised advice on when to stop ECS therapy (based on Villalta scores, see function 14a in the Limburg model) following local protocol. In North-Holland, some internists and dermatologists (dermatologists also conduct one consultation for patients with DVT at hospital location A, see function 7 in the North-Holland model) advised to stop the ECS therapy after 6 months if no complaints existed based on their most recent knowledge of literature, while others advised to stop ECS after 2 years according to the local protocol. Internists and dermatologists in North-Holland acknowledged that they were not aware of each other's instructions. As a result, patients in North-Holland mentioned that they received different advice regarding the duration of ECS therapy from dermatologists and internists. Moreover, as the moment of ECS therapy to be stopped generally takes place after follow-up appointments have ended, and no healthcare professionals were involved in this decision anymore, patients mentioned that it was especially difficult for them to understand the correct duration of therapy. It was stated that a longer follow-up duration and a more uniform, personalised method to substantiate the advice when to stop ECS therapy are necessary for patients with DVT.

\section{DISCUSSION}

This study provides important insight into the complex structure of the organisation of ECS therapy for patients with DVT and CVI in daily practice in the Netherlands, from a multiple stakeholder perspective. By using FRAM, we were able to identify the essential functions of ECS therapy. Since the majority of essential functions were equal for patients with DVT and CVI, especially for phase 1 (the initial compression therapy), phase 2 (initiating ECS therapy) and phase 3 (definitive ECS therapy), we were able to merge the processes and developed two models (one per region). Based on these models, we identified major sources of variability in the performance of the different functions and assessed the influence of variability on outcomes for patient care in discussion with the stakeholders. This ultimately resulted in five themes for improvement: dissemination of knowledge of the entire process among all healthcare professionals involved; optimising and standardising initial compression therapy; optimising timing to contact the medical stocking supplier (when oedema has disappeared); improving the implementation of assistive devices to maintain patient's self-reliance; harmonising follow-up duration for patients with CVI, and personalising follow-up and treatment duration in patients with DVT.

Previous studies on the implementation of improvement interventions have mainly focused on the individual (single component) performances instead of focusing on 'a system approach'. A review by Lau et al revealed that, in general, such strategies directed at individual professionals only achieved a small to modest improvement (range $2 \%-9 \%$ ) compared with no strategy. ${ }^{55}$ It is suggested that the use of a system approach, focusing on incorporating an understanding of how systems work as a whole from different perspectives, could facilitate implementation of improvement interventions. ${ }^{56}$

The large variation in the advised duration of therapy for patients with DVT observed in our study is consistent with data from previous studies, although none of the studies was performed in the Netherlands. Kahn et al showed that $38 \%$ of physicians recommended ECS to be worn until symptoms improved, $18 \%$ recommend it to be 
worn for a defined period, $26 \%$ recommended its indefinite use and $18 \%$ provided other advice regarding ECS therapy duration..$^{28}$ Roche-Nagle et al also highlighted that there is a large variety in the advised duration of therapy (ranging from 1 month to 2 years).${ }^{13}$ Additionally, the OTIS-DVT trial showed variable duration of therapy ranging from a median of 92 days in private practices to 364 days in patients treated in hospitals. ${ }^{33}$

Furthermore, our results emphasise the importance of maintaining patient's self-reliance for both initial compression therapy and definitive ECS therapy. We identified that, for the medical stocking supplier, time pressure, the reimbursement system of insurance companies, and the cluster fee received are barriers to optimally select and implement assistive devices for definitive ECS. We hypothesise that these barriers result in an excess of patients receiving home care, with major consequences for the patient's self-reliance, home care burden and healthcare costs. An earlier study of Sippel $e t$ al showed that the use of an assistive device could increase the ability of elderly patients with CVI to apply the ECS (closed-toe, pressure $40 \mathrm{~mm} \mathrm{Hg}$ ) self-reliantly from $60 \%$ to $90 \%,{ }^{30}$ which again emphasises the importance of optimal selection and implementation of assistive devices. Future studies should focus on creating transparency in healthcare costs regarding the implementation of assistive devices, and improvement interventions to optimise this care.

Additionally, our results showed the importance of dissemination of knowledge and skills among the different healthcare professionals involved in ECS therapy. The degree of knowledge about the process in its entirety was perceived to have strong implications for the alignment of functions in the process. This is in line with previous findings that sharing knowledge and interaction between different professionals is essential to deliver high-quality patient care in complex contexts. ${ }^{57}$

With this study, we provided a detailed insight into how ECS therapy care is organised and delivered in daily practice for all patients receiving ECS therapy. The major strength of this study is the interdisciplinary and interorganisational design, which shows the multicentre applicability of the FRAM providing the possibility to compare challenges and strengths across different settings. Furthermore, the study enabled a first insight into the reproducibility of the FRAM between regions, which is an area that is not yet extensively explored. The large number of interviewees included from each professional group and the participation of stakeholders from all groups in the discussion meetings are also strengths of the current study. This made it possible to integrate different perspectives and to provide a detailed understanding of the process in its entirety including the six themes for improvement.

However, it is possible that with more participants, more variability could have been identified, providing a wider range of perspectives for improvement interventions even though data saturation appeared to be achieved and the models and discussion points were presented and validated by all stakeholders involved in the process. Additionally, due to COVID-19, we were forced to perform several interviews by telephone, which prevented us from interviewing the participants in their working context, which could have provided further understanding of specific working conditions. Furthermore, since the interviews were conducted by a single researcher (RS) with a medical background, the possibility of observer bias arises. Pre-understanding of the context may have advanced data collection, but it might also have influenced how the interviews were conducted. We tried to control this bias by discussing the content of the interviews with also nonmedical coauthors to incorporate different views into the interpretation of the results.

\section{CONCLUSION}

In this study conducted among multiple stakeholders, we used the FRAM to integrate different perspectives and to provide a detailed insight into how the complex process of ECS therapy is delivered in daily practice in the Netherlands. We identified six major themes for improvement, which can be used to direct future intervention strategies. Furthermore, these themes can also be used as a foundation for a general cross-domain protocol for all healthcare professionals involved.

\section{Author affiliations}

${ }^{1}$ Department of Internal Medicine, Maastricht University Medical Centre+, Maastricht, The Netherlands

${ }^{2}$ Cardiovascular Research Institute Maastricht (CARIM), Maastricht University, Maastricht, The Netherlands

${ }^{3}$ Department of Clinical Epidemiology and Medical Technology Assessment (KEMTA), Maastricht University Medical Centre+, Maastricht, The Netherlands

${ }^{4}$ Heart and Vascular Center and Thrombosis Expertise Center, Maastricht University Medical Centre+, Maastricht, The Netherlands

Contributors Study design—RS, MJ and AJtC-H. Interviews—RS. Data analysis, interpretation and guidance of stakeholder meetings- RS, MJ and AJtC-H. Manuscript writing and revision—RS, MJ, AJtC-H and HTC. The manuscript has been read and approved for submission to JTH by all authors.

Funding This work was supported by ZonMW, the Netherlands Organisation for Health Research and Development (a government organisation promoting research into the cost-effectiveness of medical treatments) (grant number 84300095003).

Competing interests None declared.

Patient consent for publication Not required.

Ethics approval This study was assessed by the Ethics Board of Maastricht University Medical Center (MUMC), Maastricht (2019-1125), and was considered not to be subject to the Medical Research involving Human Subjects Act (WMO).

Provenance and peer review Not commissioned; externally peer reviewed.

Data availability statement All data relevant to the study are included in the article or uploaded as supplemental information. There are no contradictory statements

Supplemental material This content has been supplied by the author(s). It has not been vetted by BMJ Publishing Group Limited (BMJ) and may not have been peer-reviewed. Any opinions or recommendations discussed are solely those of the author(s) and are not endorsed by BMJ. BMJ disclaims all liability and responsibility arising from any reliance placed on the content. Where the content includes any translated material, BMJ does not warrant the accuracy and reliability of the translations (including but not limited to local regulations, clinical guidelines, 
terminology, drug names and drug dosages), and is not responsible for any error and/or omissions arising from translation and adaptation or otherwise.

Open access This is an open access article distributed in accordance with the Creative Commons Attribution Non Commercial (CC BY-NC 4.0) license, which permits others to distribute, remix, adapt, build upon this work non-commercially, and license their derivative works on different terms, provided the original work is properly cited, appropriate credit is given, any changes made indicated, and the use is non-commercial. See: http://creativecommons.org/licenses/by-nc/4.0/.

ORCID iD

Rachel Hellen Petra Schreurs http://orcid.org/0000-0003-3955-867X

\section{REFERENCES}

1 Prandoni P, Lensing AWA, Prins MH, et al. Below-knee elastic compression stockings to prevent the post-thrombotic syndrome: a randomized, controlled trial. Ann Intern Med 2004;141:249-56.

2 Brandjes DP, Büller HR, Heijboer H, et al. Randomised trial of effect of compression stockings in patients with symptomatic proximal-vein thrombosis. Lancet 1997;349:759-62.

3 Mol GC, van de Ree MA, Klok FA, et al. One versus two years of elastic compression stockings for prevention of post-thrombotic syndrome (OCTAVIA study): randomised controlled trial. BMJ 2016;353:i2691.

4 Ten Cate-Hoek AJ, Amin EE, Bouman AC, et al. Individualised versus standard duration of elastic compression therapy for prevention of post-thrombotic syndrome (ideal DVT): a multicentre, randomised, single-blind, allocation-concealed, non-inferiority trial. Lancet Haematol 2018;5:e25-33.

5 Wittens C, Davies AH, Bækgaard N, et al. Editor's choice management of chronic venous disease: clinical practice guidelines of the european society for vascular surgery (ESVS). Eur J Vasc Endovasc Surg 2015;49:678-737.

6 Rabe E, Partsch H, Hafner J, et al. Indications for medical compression stockings in venous and lymphatic disorders: an evidence-based consensus statement. Phlebology 2018;33:163-84.

7 Berszakiewicz A, Sieroń A, Krasiński Z, et al. Compression therapy in venous diseases: current forms of compression materials and techniques. Postepy Dermatol Alergol 2020;37:836-41.

8 Berszakiewicz A, Sieron A, Krasiński Z, et al. Compression therapy in venous diseases: physical assumptions and clinical effects. Postepy Dermatol Alergol 2020;37:842-7.

9 Dahm KT, Myrhaug HT, Strømme H, et al. Effects of preventive use of compression stockings for elderly with chronic venous insufficiency and swollen legs: a systematic review and meta-analysis. BMC Geriatr 2019;19:76.

10 Raju S, Hollis K, Neglen P. Use of compression stockings in chronic venous disease: patient compliance and efficacy. Ann Vasc Surg 2007;21:790-5.

11 Kankam HKN, Lim CS, Fiorentino F, et al. A summation analysis of compliance and complications of compression hosiery for patients with chronic venous disease or post-thrombotic syndrome. Eur $J$ Vasc Endovasc Surg 2018;55:406-16.

12 Dawson AJ, Akaberi A, Galanaud J-P, et al. Patient-reported reasons for and predictors of noncompliance with compression stockings in a randomized trial of stockings to prevent postthrombotic syndrome. Res Pract Thromb Haemost 2020;4:269-77.

13 Roche-Nagle G, Ward F, Barry M. Current prescribing patterns of elastic compression stockings post-deep venous thrombosis. Phlebology 2010;25:72-8.

14 Bouman AC, Ten Cate-Hoek AJ, Dirksen CD, et al. Eliciting patients preferences for elastic compression stocking therapy after deep vein thrombosis: potential for improving compliance. J Thromb Haemost 2016;14:510-7.

15 Carolyn C. Post-thrombotic syndrome patient education based on the health belief model: self-reported intention to comply with recommendations. J Wound Ostomy Continence Nurs 2011;38:648-54.

16 Gong J-M, Du J-S, Han D-M, et al. Reasons for patient noncompliance with compression stockings as a treatment for varicose veins in the lower limbs: a qualitative study. PLoS One 2020;15:e0231218.

17 Reich-Schupke S, Murmann F, Altmeyer P, et al. Compression therapy in elderly and overweight patients. Vasa 2012;41:125-31.

18 Amin EE, Ten Cate-Hoek AJ, Bouman AC, et al. Individually shortened duration versus standard duration of elastic compression therapy for prevention of post-thrombotic syndrome: a costeffectiveness analysis. Lancet Haematol 2018;5:e512-9.
19 The Health Foundation. Evidence scan: complex adaptive systems. London: The Health Foundation, 2010.

20 Plsek PE, Greenhalgh T. Complexity science: the challenge of complexity in health care. BMJ 2001;323:625-8.

21 Hollnagel E. FRAM: the functional resonance analysis method: modelling complex socio-technical systems, 2012.

22 Rubenstein LV, Pugh J. Strategies for promoting organizational and practice change by advancing implementation research. $J$ Gen Intern Med 2006;21 Suppl 2:S58-64

23 Murphy GJ, Reeves BC, Rogers CA, et al. Increased mortality, postoperative morbidity, and cost after red blood cell transfusion in patients having cardiac surgery. Circulation 2007;116:2544-52.

24 May CR, Johnson M, Finch T. Implementation, context and complexity. Implement Sci 2016:11:141.

25 Campbell NC, Murray E, Darbyshire J, et al. Designing and evaluating complex interventions to improve health care. BMJ 2007;334:455-9.

26 Kahn SR, Shapiro S, Wells PS, et al. Compression stockings to prevent post-thrombotic syndrome: a randomised placebo-controlled trial. Lancet 2014;383:880-8.

27 Azirar S, Appelen D, Prins MH, et al. Compression therapy for treating post-thrombotic syndrome. Cochrane Database Syst Rev 2019;9:CD004177.

28 Kahn SR, Elman E, Rodger MA, et al. Use of elastic compression stockings after deep venous thrombosis: a comparison of practices and perceptions of thrombosis physicians and patients. J Thromb Haemost 2003;1:500-6.

29 Berszakiewicz A, Kasperczyk J, Sieron A, et al. The effect of compression therapy on quality of life in patients with chronic venous disease: a comparative 6-month study. Postepy Dermatol Alergol 2021;38:389-95

30 Sippel K, Seifert B, Hafner J. Donning devices (foot slips and frames) enable elderly people with severe chronic venous insufficiency to put on compression stockings. Eur J Vasc Endovasc Surg 2015;49:221-9.

31 Mosti G, Cavezzi A, Partsch H, et al. Adjustable velcro compression devices are more effective than inelastic bandages in reducing venous edema in the initial treatment phase: a randomized controlled trial. Eur J Vasc Endovasc Surg 2015;50:368-74.

32 Gelderblom G, Hagedoorn-Meuwissen EAV. Easy-Lever Kuittrekhulpmiddel Een Onderzoek naar bruikbaarheid. effecten en belemmeringen 2005.

33 Spirk D, Banyai M, Jacomella V, et al. Outpatient management of acute deep vein thrombosis: results from the OTIS-DVT registry. Thromb Res 2011;127:406-10.

34 Dua A, Desai SS, Heller JA. Heterogeneity in venous disease practice patterns amongst primary healthcare practitioners. Vascular 2015;23:391-5.

35 Hollnagel E. Safety-I and safety-II. The past and future of safety management. Farnham, UK: Ashgate Publishing Limited, 2014.

36 Damen NL, de Vos MS, Moesker MJ. Preoperative anticoagulation management in everyday clinical practice: an international comparative analysis of work-as-done using the functional resonance analysis method. $J$ Patient Saf 2018.

37 Clay-Williams R, Hounsgaard J, Hollnagel E. Where the rubber meets the road: using FRAM to align work-as-imagined with workas-done when implementing clinical guidelines. Implement Sci 2015;10:125.

$38 \mathrm{McNab}$ D, Freestone J, Black C, et al. Participatory design of an improvement intervention for the primary care management of possible sepsis using the functional resonance analysis method. BMC Med 2018;16:174.

39 Raben DC, Bogh SB, Viskum B, et al. Learn from what goes right: a demonstration of a new systematic method for identification of leading indicators in healthcare. Reliability Engineering \& System Safety 2018;169:187-98.

40 O'Hara JK, Baxter R, Hardicre N. 'Handing over to the patient': a FRAM analysis of transitional care combining multiple stakeholder perspectives. Appl Ergon 2020;85:103060.

41 Laugaland K, Aase K, Waring J. Hospital discharge of the elderly--an observational case study of functions, variability and performanceshaping factors. BMC Health Serv Res 2014;14:365.

42 Furniss D, Nelson D, Habli I, et al. Using FRAM to explore sources of performance variability in intravenous infusion administration in ICU: a non-normative approach to systems contradictions. Appl Ergon 2020;86:103113.

43 Schutijser BCFM, Jongerden IP, Klopotowska JE, et al. Double checking injectable medication administration: does the protocol fit clinical practice? Saf Sci 2019;118:853-60.

44 Pickup L, Atkinson S, Hollnagel E, et al. Blood sampling - two sides to the story. Appl Ergon 2017;59:234-42. 
45 Kaya GK, Ovali HF, Ozturk F. Using the functional resonance analysis method on the drug administration process to assess performance variability. Saf Sci 2019;118:835-40.

46 Buikstra E, Strivens E, Clay-Williams R. Understanding variability in discharge planning processes for the older person. Saf Sci 2020;121:137-46.

47 Eklöf B, Rutherford RB, Bergan JJ, et al. Revision of the CEAP classification for chronic venous disorders: consensus statement. $J$ Vasc Surg 2004;40:1248-52.

48 Hill R. FRAM model visualizer version 0.4.1. Available: http:// functionalresonance.com/the $\% 20$ fram $\% 20$ model\%20visualiser.html

49 Hollnagel E, Hounsgaard J, Colligan L. FRAM- the functional resonance analysis method - a handbook for practical use of the method, 2014. Available: https://functionalresonance.com/ onewebmedia/FRAM handbook_web-2.pdf

50 Kwaliteitsinstituut voor de Gezondheidszorg CBO. CBO richtlijn diagnostiek preventie en behandeling van veneuze trombo-embolie en secundaire preventie van arteriele trombose, 2008. Available: https://www.nvr.nl/cbo-richtlijnen

51 Nederlands Huisartsen Genootschap. NHG-standaard diepveneuze trombose en longembolie, 2017. Available: https://richtlijnen.nhg.org/ standaarden/diepveneuze-trombose-en-longembolie [Accessed 17 Sep 2020].

52 Genootschap NH. NHG standaard varices, 2009. Available: https:// richtlijnen.nhg.org/standaarden/varices [Accessed 17 Sep 2020]

53 Nederlandse Vereniging voor Dermatologie en Venereologie (NVDV). Richtlijn veneuze pathologie, 2014. Available: https://www. huidziekten.nl/richtlijnen/richtlijn-veneuze-pathologie-2014.pdf [Accessed 17 Sep 2020].

54 Mason M. Sample size and saturation in PHD studies using qualitative interviews. Available: http://www.qualitative-research.net/ index.php/fqs/article/view/14282010 [Accessed 17 Sep 2020].

55 Lau R, Stevenson F, Ong BN, et al. Achieving change in primary care--effectiveness of strategies for improving implementation of complex interventions: systematic review of reviews. BMJ Open 2015;5:e009993.

56 Greenhalgh T, Papoutsi C. Studying complexity in health services research: desperately seeking an overdue paradigm shift. BMC Med 2018;16:95

57 Nancarrow SA, Booth A, Ariss S, et al. Ten principles of good interdisciplinary team work. Hum Resour Health 2013;11:19. 